\title{
Atenção plena e aconselhamento nutricional na promoção da consciência do comportamento alimentar: revisão integrativa
}

\author{
Mindfulness and nutritional counseling in promoting awareness of eating behavior: integrative \\ review \\ Atención plena y asesoramiento nutricional en la promoción de la conciencia de la conducta \\ alimentaria: revisión integradora
}

\author{
Mayara Priscilla Dantas Araújo \\ ORCID: https://orcid.org/0000-0002-0611-2949 \\ Universidade Federal do Rio Grande do Norte, Brasil \\ E-mail: larissagrace.nutricionista@gmail.com \\ Raísa Acácio França Costa \\ ORCID: https://orcid.org/0000-0001-9809-3716 \\ Universidade Federal do Rio Grande do Norte, Brasil \\ E-mail: raisaacaciofranca@gmail.com \\ Larissa Grace Serafim Nogueira de Melo \\ ORCID: https://orcid.org/0000-0003-4204-5517 \\ Universidade Federal do Rio Grande do Norte, Brasil \\ E-mail: larissagrace.nutricionista@gmail.com
}

\begin{abstract}
Resumo
Objetivo: Identificar a eficácia da atenção plena e do aconselhamento nutricional na promoção do comportamento alimentar. Método: Trata-se de uma revisão integrativa, realizada entre os meses de agosto e setembro de 2020, com buscas nas bases de dados BVS e PubMed. Foram utilizados os descritores: atenção plena, comportamento alimentar e adulto, em inglês, português e espanhol. Resultados: sete artigos compuseram a amostra do estudo. Todos os estudos realizaram intervenções de atenção plena associada ao aconselhamento nutricional. Os resultados obtidos demonstram a eficácia dessa intervenção no comportamento e escolhas alimentares, na redução de peso em paciente obesos, contribui para melhoria da qualidade de vida e promover uma maior consciência alimentar. As intervenções obtiveram alta aceitação. Considerações finais: A prática de atenção plena associada ao aconselhamento nutricional se mostrou eficaz para promoção da consciência alimentar e adoção de um comportamento alimentar mais saudável, trazendo inúmeros benefícios para a qualidade de vida daqueles que a praticam.
\end{abstract}

Palavras-chave: Atenção plena; Comportamento alimentar; Promoção da saúde.

\begin{abstract}
Objective: To identify the effectiveness of mindfulness and nutritional counseling in promoting eating behavior. Method: This is an integrative review, carried out between August and September 2020, with searches in the BVS and Pubmed databases. The following descriptors were used: mindfulness, eating behavior and adult, in English, Portuguese and Spanish. Results: seven articles composed the study sample. All studies performed interventions of mindfulness associated with nutritional counseling. The results obtained demonstrate the effectiveness of this intervention in the behavior and dietary choices, in reducing weight in obese patients, contributes to improve the quality of life and promote greater food awareness. The interventions obtained high acceptance. Final considerations: The practice of mindfulness associated with nutritional counseling proved effective to promote food awareness and adopt a healthier eating behavior, bringing numerous benefits to the quality of life of those who practice it.
\end{abstract}

Keywords: Mindfulness; Feeding behavior; Health promotion.

\section{Resumen}

Objetivo: Identificar la eficacia de la atención plena y del asesoramiento nutricional en la promoción del comportamiento alimentario. Método: Se trata de una revisión integradora, realizada entre los meses de agosto y septiembre de 2020, con búsquedas en las bases de datos BVS y PubMed. Se utilizaron los siguientes descriptores: atención plena, conducta alimentaria y adulto, en inglés, portugués y español. Resultados: La muestra del estudio estaba compuesta por siete artículos. Todos los estudios realizaron intervenciones de mindfulness asociadas al asesoramiento nutricional. Los resultados obtenidos demuestran la eficacia de esta intervención en el comportamiento y la elección de alimentos, la reducción de peso en pacientes obesos, contribuyendo a mejorar la calidad de vida y promoviendo una mayor conciencia alimentaria. Las intervenciones obtuvieron una gran aceptación. Consideraciones 
finales: La práctica de la atención plena asociada al asesoramiento nutricional se muestra eficaz para la promoción de la conciencia alimentaria y la adopción de un comportamiento alimentario más saludable, aportando numerosos beneficios para la calidad de vida de quienes la practican.

Palabras clave: Atención plena; Conducta alimentaria; Promoción de la salud.

\section{Introdução}

A atenção plena é uma prática e uma forma de consciência que tem sido utilizada como base para intervenções no cuidado e promoção da saúde (Hirayama, et al., 2014). Intervenções baseadas na atenção plena são consideradas práticas integrativas de cuidado e trazem inúmeros benefícios para o indivíduo, que pode ser ensinada e/ou aprendida por qualquer pessoa sem trazer efeitos adversos à saúde (Lucas, et al., 2017).

Essas intervenções, quando realizadas no âmbito da alimentação e nutrição, podem ser eficazes na redução da compulsão alimentar, do comer emocional e das preocupações com a imagem corporal e mostraram resultados promissores para o controle de peso, estando associada a uma maior consciência alimentar (O’Reilly, et al., 2014).

A atenção plena ao comer busca estreitar a relação do indivíduo com o alimento, de forma que o alimento não seja um problema e leve a disfunções no comportamento alimentar, como o comer emocional e excessivo. Busca-se, então, a consciência alimentar, que consiste em fazer escolhas alimentares de forma consciente e com maior atenção, compreendendo os aspectos psicológicos que interferem na alimentação e no ato de se alimentar, contribuindo para uma alimentação e comportamento alimentar mais saudáveis (Warren, et al., 2017).

Essa prática vem como uma alternativa para os tratamentos de primeira linha para intervenções no estilo de vida, estando associada a uma maior autoeficácia em relação a dieta, perda de peso e, até mesmo, prática de atividade física (Mason, Epel, Kristeller, et al., 2016). As intervenções tradicionais, principalmente quanto a perda de peso, levam a exacerbação de fatores psicológicos, resultando em uma alimentação excessiva e por recompensa. Além do mais, o ambiente alimentar moderno é um desafio por si só, estimulando o comer excessivo como consequência do comer de forma inconsciente e automática (Mason, Epel, Aschbacher, et al., 2016). Dessa forma, buscou-se identificar, a partir da literatura, a eficácia das intervenções baseadas na atenção plena associadas ao aconselhamento nutricional na promoção do comportamento alimentar saudável.

\section{Metodologia}

O presente estudo trata-se de uma revisão integrativa, método de pesquisa que permite a síntese e avaliação crítica de estudos de diferentes abordagens metodológicas, possibilitando a criação de um panorama consistente para compreensão de conceitos, teorias e problemas de saúde, fundamentadas em evidências científicas e que contribuem para a prática clínica (Souza, et al., 2010).

Para esta revisão foram adotadas as seguintes etapas, conforme Mendes et al. (2008): (1) identificação do tema e seleção da pergunta norteadora; (2) estabelecimento de critérios de inclusão e exclusão; (3) definição das informações a serem extraídas dos estudos; (4) avaliação dos estudos; (5) interpretação dos resultados; e (6) síntese do conhecimento.

Esta revisão objetivou responder à seguinte questão: a associação de intervenções baseadas na atenção plena com aconselhamento nutricional é eficaz para promoção da consciência do comportamento alimentar saudável em pessoas adultas?

A seleção dos artigos foi realizada entre os meses de agosto e setembro de 2020. Foram realizadas buscas nas bases de dados eletrônicas Biblioteca Virtual em Saúde (BVS) e U.S. National Library of Medicine (PubMed). Para as buscas, foram utilizados descritores indexados segundo a padronização dos Descritores em Ciências da Saúde (DeCS) e MeSH (Medical Subject Headings) nas versões em português, inglês e espanhol. Os descritores utilizados foram: atenção plena (mindfulness, atención plena), comportamento alimentar (feeding behavior, conducta alimentaria) e adulto (adult, adulto). Esses descritores 
foram combinados através do uso dos operadores booleanos AND e OR, sendo utilizadas as seguintes combinações nas buscas: [(atenção plena) AND (comportamento alimentar) AND (adulto)], [(mindfulness) AND (feeding behavior) AND (adult)] e [(atención plena) AND (conducta alimentaria) AND (adulto)].

Foram estabelecidos os seguintes critérios de inclusão: estudos originais publicados entre janeiro de 2016 e agosto de 2020, nos idiomas português, inglês ou espanhol, disponíveis gratuitamente para leitura na íntegra, independentemente do número de indivíduos estudados e que abordassem plena ou parcialmente o conteúdo. Foram excluídos estudos que abordassem outros ciclos da vida (crianças, gestantes e idosos), materiais como livros, capítulos de livros, monografias, editoriais, cartas de apresentação, resenhas, resumos em anais de congressos, comentários e artigos de revisão de literatura.

Para identificar se os estudos encontrados atendiam aos critérios estabelecidos para esta revisão, inicialmente foram identificados os estudos duplicados, os quais foram computados apenas uma vez, em seguida, foi realizada a leitura dos títulos e resumos considerando os critérios de elegibilidade. Os artigos selecionados nesta etapa foram recuperados para leitura na íntegra, sendo aplicado novamente os critérios de inclusão e exclusão durante a leitura do texto completo. Após análise do texto completo, os artigos que contemplaram todos os critérios compuseram a amostra final deste estudo.

Para a etapa de análise, foi realizado o fichamento dos artigos utilizando planilha eletrônica do Microsoft Excel®, de forma a sintetizar e organizar os dados. Para extração dos dados, foram considerados os seguintes itens: título, autores, ano de publicação, país em que o estudo foi desenvolvido, delineamento do estudo, amostra, intervenção utilizada e desfecho/conclusão.

\section{Resultados}

As buscas nas bases de dados resultaram em 82 publicações, das quais 53 foram oriundas da BVS e 29 da PubMed. Foram identificados 17 artigos duplicados. Após leitura do título, uma publicação foi excluída por ser livro e 22 por serem estudos com indivíduos em outros ciclos de vida. Com isso, 42 publicações foram selecionadas para leitura exploratória dos resumos. Destas, 18 foram excluídas por não apresentarem intervenção ou não estarem relacionadas à temática estudada. Dos 24 artigos selecionados para leitura na íntegra, sete estavam indisponíveis para leitura completa e 10 artigos foram excluídos por não responderem à questão norteadora. Ao final, sete artigos compuseram a amostra desta revisão (Figura 1). 
Figura 1. Fluxograma de seleção dos estudos.

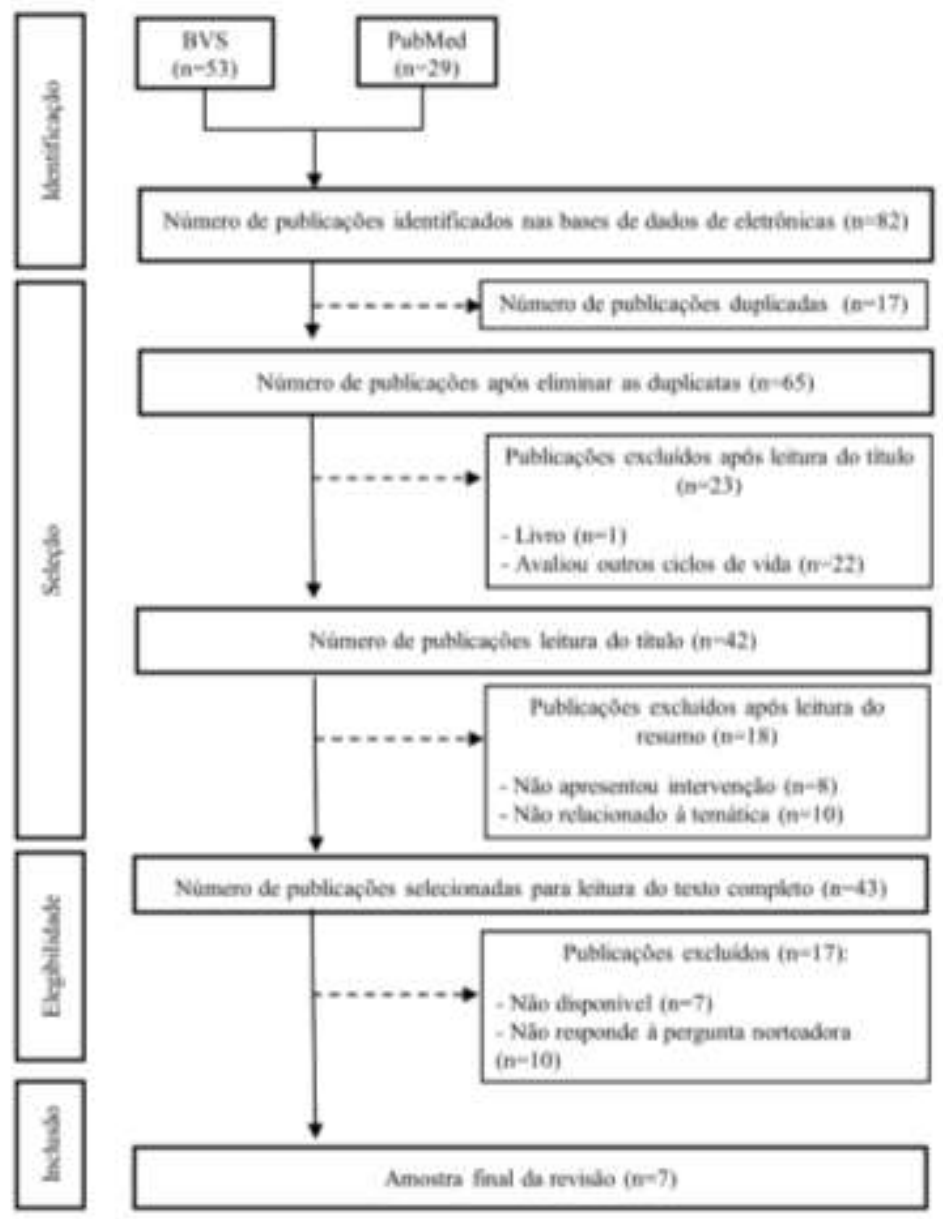

Fonte: Autores (2020).

Dos estudos que compuseram a amostra, 57,1\% $(\mathrm{n}=4)$ foram publicados em 2016. Quanto ao desenho dos estudos, $57,1 \%(n=4)$ são ensaios clínicos, 28,6\% (n=2) são estudos quase-experimentais e 14,3\% (n=1) estudos observacionais.

Os estudos apresentaram variações quanto ao tamanho amostral, sendo possível dividi-los em amostras com menos de 20 participantes (Chacko, et al., 2016; Lucas, et al., 2017), com até 100 participantes (Gidugu \& Jacobs, 2019; Hanson, et al., 2019; Palmeira, et al., 2017) e com mais de 100 participantes (Mason, Epel, Aschbacher, et al., 2016; Mason, Epel, Kristeller, et al., 2016).

Considerando as características das amostras avaliadas, quatro artigos envolveram apenas indivíduos obesos, destes, dois foram realizados nos Estados Unidos e utilizaram a mesma amostra, diferenciados pelo momento em que os participantes foram avaliados (Mason, Epel, Aschbacher, et al., 2016; Mason, Epel, Kristeller, et al., 2016), um estudo foi realizado com pacientes de um serviço de controle de peso de um hospital universitário no Reino Unido (Hanson, et al., 2019) e um realizado apenas com indivíduos do sexo feminino em Portugal (Palmeira, et al., 2017). Quantos aos demais estudos selecionados, um envolveu apenas pacientes bariátricos (Chacko, et al., 2016), um avaliou apenas mulheres com câncer de endométrio com sobrepeso (Lucas, et al., 2017) e um foi realizado com pacientes com doença mental grave (Gidugu \& Jacobs, 2019) (Quadro $1)$. 
Quadro 1. Caracterização dos artigos selecionados.

\begin{tabular}{|c|c|c|c|c|c|}
\hline $\begin{array}{l}\text { Autores, ano e } \\
\text { país do estudo }\end{array}$ & $\begin{array}{l}\text { Desenho do } \\
\text { estudo }\end{array}$ & Objetivo & Amostra & $\begin{array}{l}\text { Intervenção/ } \\
\text { observação }\end{array}$ & Desfechos \\
\hline $\begin{array}{l}\text { Chacko et al. } \\
2016 \\
\text { Estados Unidos }\end{array}$ & $\begin{array}{l}\text { Ensaio piloto } \\
\text { randomizado e } \\
\text { controlado }\end{array}$ & $\begin{array}{l}\text { Desenvolver e testar uma } \\
\text { nova IBM projetada para } \\
\text { controlar o peso após a } \\
\text { cirurgia bariátrica }\end{array}$ & $\begin{array}{l}18 \text { adultos com } 1 \text { a } 5 \\
\text { anos pós-bariátrica e } \\
\text { com perda de peso <5 } \\
\text { libras nos últimos } 3 \\
\text { meses (Intervenção } \\
\text { n=9 e controle } \mathrm{n}=9 \text { ) }\end{array}$ & $\begin{array}{l}\text { Comparação da } \\
\text { IBM com } \\
\text { intervenção padrão } \\
\text { durante } 10 \\
\text { semanas }\end{array}$ & $\begin{array}{l}\text { Os resultados sugerem } \\
\text { que a intervenção pode } \\
\text { ser eficaz para reduzir o } \\
\text { comer emocional, mas } \\
\text { não o peso }\end{array}$ \\
\hline $\begin{array}{l}\text { Lucas et al. } \\
2017 \\
\text { Estados Unidos }\end{array}$ & $\begin{array}{l}\text { Estudo quase- } \\
\text { experimental }\end{array}$ & $\begin{array}{l}\text { Avaliar a viabilidade da } \\
\text { IBM associada ao } \\
\text { aconselhamento dietético } \\
\text { e sua eficácia na } \\
\text { melhoria da atenção } \\
\text { plena, dieta, atividade } \\
\text { física e qualidade de } \\
\text { vida } \\
\end{array}$ & $\begin{array}{c}17 \text { mulheres com } \\
\text { diagnóstico prévio de } \\
\text { câncer de endométrio } \\
\text { de grau } 1 \text { ou } 2, \text { estágio } \\
\text { I ou II, com IMC > } 25 \\
\text { e sedentárias }\end{array}$ & $\begin{array}{l}\text { Intervenção de } 8 \\
\text { semanas de } \\
\text { aconselhamento } \\
\text { dietético e IBM }\end{array}$ & $\begin{array}{l}\text { Não foi observado } \\
\text { nenhum resultado } \\
\text { significativo }\end{array}$ \\
\hline $\begin{array}{l}\text { Mason, Epel, } \\
\text { Kristeller, et al. } \\
2016 \\
\text { Estados Unidos }\end{array}$ & $\begin{array}{l}\text { Ensaio clínico } \\
\text { randomizado } \\
\text { controlado }\end{array}$ & $\begin{array}{c}\text { Avaliar o impacto de } \\
\text { mudanças na } \\
\text { alimentação consciente } \\
\text { na ingestão de alimentos } \\
\text { doces e nos níveis de } \\
\text { glicose em jejum } \\
\end{array}$ & $\begin{array}{c}194 \text { adultos obesos } \\
\left(\text { IMC }>30 \mathrm{~kg} / \mathrm{m}^{2}\right) \\
(\text { Intervenção } \mathrm{n}=100 \mathrm{e} \\
\text { controle } \mathrm{n}=94)\end{array}$ & $\begin{array}{l}\text { Dieta e exercícios } \\
\text { associados ao MB- } \\
\text { EAT com duração } \\
\text { de } 5 \text { meses e meio }\end{array}$ & $\begin{array}{c}\text { O aumento da } \\
\text { consciência alimentar } \\
\text { foi associado a } \\
\text { diminuições na ingestão } \\
\text { de doces e da glicemia } \\
\text { em jejum } \\
\end{array}$ \\
\hline $\begin{array}{l}\text { Mason, Epel, } \\
\text { Aschbacher, et } \\
\text { al. } \\
2016 \\
\text { Estados Unidos }\end{array}$ & $\begin{array}{l}\text { Ensaio clínico } \\
\text { randomizado } \\
\text { controlado }\end{array}$ & $\begin{array}{c}\text { Avaliar o } \\
\text { comportamento } \\
\text { alimentar e estresse } \\
\text { psicológico pré e pós- } \\
\text { intervenção e a perda de } \\
\text { peso } 12 \text { e } 18 \text { meses após } \\
\text { a intervenção }\end{array}$ & $\begin{array}{c}194 \text { adultos obesos } \\
\left(\text { IMC }>30 \mathrm{~kg} / \mathrm{m}^{2}\right) \\
(\text { Intervenção } \mathrm{n}=100 \mathrm{e} \\
\text { controle } \mathrm{n}=94)\end{array}$ & $\begin{array}{l}\text { Dieta e exercícios } \\
\text { associados ao MB- } \\
\text { EAT com duração } \\
\text { de } 5 \text { meses e meio }\end{array}$ & $\begin{array}{l}\text { O aumento da } \\
\text { consciência alimentar, } \\
\text { pela redução da } \\
\text { alimentação baseada em } \\
\text { recompensas, está } \\
\text { associado a diminuições } \\
\text { no peso a longo prazo }\end{array}$ \\
\hline $\begin{array}{l}\text { Palmeira, Pinto- } \\
\text { Gouveia e } \\
\text { Cunha } \\
2017 \\
\text { Portugal }\end{array}$ & $\begin{array}{l}\text { Ensaio clínico } \\
\text { randomizado }\end{array}$ & $\begin{array}{l}\text { Testar a eficácia do Kg- } \\
\text { Free em mulheres com } \\
\text { sobrepeso e obesidade } \\
\text { sem compulsão } \\
\text { alimentar }\end{array}$ & $\begin{array}{c}73 \text { mulheres adultas } \\
\text { com sobrepeso e } \\
\text { obesidade (IMC > } \\
\left.25 \mathrm{~kg} / \mathrm{m}^{2}\right)(\text { Intervenção } \\
\mathrm{n}=36 \text { e controle } \mathrm{n}=37)\end{array}$ & $\begin{array}{l}\text { Utilizou-se o Kg- } \\
\text { free, intervenção } \\
\text { baseada aceitação, } \\
\text { atenção e } \\
\text { compaixão }\end{array}$ & $\begin{array}{l}\text { A intervenção reduziu } \\
\text { significativamente o } \\
\text { auto estigma de peso, } \\
\text { comer emocional e } \\
\text { excessivo e aumentou a } \\
\text { qualidade de vida }\end{array}$ \\
\hline $\begin{array}{l}\text { Gidugu e Jacobs } \\
2019 \\
\text { Estados Unidos }\end{array}$ & $\begin{array}{l}\text { Estudo quase- } \\
\text { experimental }\end{array}$ & $\begin{array}{c}\text { Desenvolver um } \\
\text { programa de capacitação } \\
\text { sobre alimentação } \\
\text { consciente e educação } \\
\text { nutricional para pessoas } \\
\text { doença mental grave }\end{array}$ & $\begin{array}{l}46 \text { indivíduos com } \\
\text { doença mental grave }\end{array}$ & $\begin{array}{l}\text { Programa de } \\
\text { capacitação de } 14 \\
\text { semanas sobre } \\
\text { alimentação } \\
\text { consciente e } \\
\text { educação } \\
\text { nutricional } \\
\end{array}$ & $\begin{array}{c}\text { Os participantes } \\
\text { desenvolveram maior } \\
\text { consciência alimentar } \\
\text { com redução da comer } \\
\text { excessivo e emocional }\end{array}$ \\
\hline $\begin{array}{l}\text { Hanson et al. } \\
2019 \\
\text { Inglaterra }\end{array}$ & $\begin{array}{c}\text { Estudo } \\
\text { observacional } \\
\text { sem } \\
\text { randomização }\end{array}$ & $\begin{array}{l}\text { Demonstrar a eficácia } \\
\text { clínica da atenção plena } \\
\text { como parte de sessões de } \\
\text { grupo em um serviço } \\
\text { para obesidade }\end{array}$ & $\begin{array}{c}86 \text { adultos obesos } \\
\left(\mathrm{IMC}>35 \mathrm{~kg} / \mathrm{m}^{2}\right), \\
\text { pacientes } \\
\text { do serviço de controle } \\
\text { de peso (Intervenção } \\
\mathrm{n}=53 \text { e controle } \mathrm{n}=33 \text { ) }\end{array}$ & $\begin{array}{l}4 \text { sessões de grupo } \\
\text { sobre o controle da } \\
\text { obesidade (dieta e } \\
\text { atividade física), } \\
\text { com ênfase na } \\
\text { aplicação de } \\
\text { técnicas de atenção } \\
\text { plena }\end{array}$ & $\begin{array}{c}\text { Houve melhora } \\
\text { significativa na } \\
\text { alimentação } \\
\text { autorrelatada, maior } \\
\text { perda de peso, melhora } \\
\text { na relação com a } \\
\text { alimentação e maior } \\
\text { confiança no } \\
\text { autogerenciamento da } \\
\text { perda de peso }\end{array}$ \\
\hline
\end{tabular}

Legenda: IBM: Intervenção baseada em mindfulness; IMC: Índice de massa corporal; MB-EAT: Programa de treinamento de consciência alimentar baseado em mindfulness. Fonte: Autores (2020).

Em relação ao objetivo dos estudos, $85,7 \%(\mathrm{n}=6)$ deles buscaram avaliar os resultados de uma intervenção com base na atenção plena (mindfulness) na consciência e no comportamento alimentar utilizando-se de aconselhamento nutricional e/ou práticas de comer com atenção plena (mindful eating). Dentre os estudos, somente o realizado por Hanson et al. (2019) foi um estudo observacional, mas que apresentou objetivo semelhante aos demais.

Apenas um estudo (Gidugu \& Jacobs, 2019) não avaliou a perda de peso nos participantes após a intervenção. Embora todas as intervenções tenham como base a atenção plena, foi identificado o uso de diferentes técnicas para aplicação dessa 
prática. Dos sete estudos analisados, cinco utilizaram uma intervenção baseada na atenção plena (Chacko, et al., 2016; Hanson, et al., 2019; Lucas, et al., 2017; Mason, Epel, Aschbacher, et al., 2016; Mason, Epel, Kristeller, et al., 2016), um utilizou um programa de comer com atenção plena (Gidugu \& Jacobs, 2019) e um utilizou a técnica Kg-free (Palmeira, et al., 2017).

\section{Discussão}

A atenção plena é compreendida como o direcionamento da consciência, de forma proposital, para o momento presente, sem julgamentos. Esta prática pode ser aplicada a diferentes cenários clínicos, com benefícios para o funcionamento mental e físico do organismo (Dunn, et al., 2018; Hanson, et al., 2019), além de ser uma estratégia muito utilizada na mudança de comportamento e padrões alimentares. Com base na análise dos estudos incluídos nesta revisão, identificou-se que a atenção plena, quando associada ao aconselhamento nutricional, pode influenciar de forma positiva o comportamento alimentar de indivíduos adultos com diferentes condições de saúde.

Estudos observaram que pessoas adultas com obesidade (Mason, Epel, Aschbacher, et al., 2016) e mulheres obesas com câncer de mama (Chung, et al., 2016) desenvolveram maior consciência alimentar e apresentaram perda de peso a longo prazo após intervenções de atenção plena e aconselhamento nutricional. Estes achados são corroborados por estudo que encontrou correlação negativa entre o comer com atenção plena e medidas antropométricas (Özkan \& Bilici, 2021). Isto demonstra que a maior consciência alimentar está associada a perda de peso, afirmando os benefícios e a relevância desta prática como estratégia para redução do peso em pessoas com obesidade.

Outro benefício decorrente desta prática é a maior atenção dada ao processo alimentar. Melhorias no comportamento alimentar foram identificadas através de escolhas alimentares mais saudáveis, moderação da quantidade de alimento consumido e planejamento alimentar (Chacko, et al., 2016; Hussain, et al., 2021). A atenção plena e o aconselhamento nutricional resultaram em melhora no padrão alimentar autorrelatado de pessoas com obesidade, principalmente pela diminuição do consumo de fast food (Hanson, et al., 2019), tendência de escolha por refeições mais saudáveis (Stites, et al., 2015) e redução do consumo calórico (Jordan, et al., 2014), demonstrando que a atenção plena promove a adoção de uma alimentação mais saudável, principalmente quando combinada ao aconselhamento nutricional.

Embora a atenção plena ao comer contribua para escolhas alimentares mais saudáveis através da maior atenção ao comer, em estudo realizado com mulheres com câncer de endométrio com sobrepeso e obesidade, não foram observadas mudanças significativas na qualidade da dieta das participantes após a intervenção (Lucas, et al., 2017). Porém, este estudo enfatizou, na intervenção nutricional, a redução do tamanho de porções, do consumo calórico e na perda de peso, e não a promoção do comer com atenção plena, o que poderia justificar os resultados encontrados, uma vez que as intervenções voltadas para promoção da consciência alimentar parecem ter efeito comportamental prolongado, auxiliando na manutenção do peso, importante fator para intervenções voltadas para a perda de peso e que é negligenciado (Chung, et al., 2016).

A relação disfuncional com a alimentação, como a exacerbação de controle sobre a alimentação, restrições alimentares severas e comportamento voltados para perda de peso, coocorrem com a compulsão alimentar, reduzindo a percepção sobre a quantidade de alimentos ingeridos pelo indivíduo (Oliveira, et al., 2020). Como resultados das intervenções de atenção plena e aconselhamento nutricional, foram identificadas melhoria na relação com a alimentação (Hanson, et al., 2019) e aumento da autocompaixão e da qualidade de vida (Palmeira, et al., 2017). Esses resultados demonstram que esta prática pode auxiliar na redução do comer compulsivo, atuando até mesmo como fator de proteção para relação disfuncional com a comida. Dessa forma, enfatiza-se a importância da adoção de intervenções baseadas na atenção plena para promoção de comportamentos alimentares mais saudáveis.

A redução do comer excessivo é um dos principais resultados da prática da atenção plena ao comer, conforme observado em diferentes estudos (Gidugu \& Jacobs, 2019; Palmeira, et al., 2017; Warren, et al., 2017). Isto se deve a maior 
consciência dos sinais físicos do corpo, como o reconhecimento do nível de fome e saciedade, atenção ao ritmo do comer, características do ambiente e do alimento (Dunn, et al., 2018), que auxilia no controle do consumo excessivo de alimentos e no consumo daqueles de alto teor calórico. Outra explicação é que a atenção plena está negativamente associada a impulsividade, fazendo com que a maior consciência ao momento presente diminua a impulsividade e, consequentemente, o comer excessivo e emocional (Bal, et al., 2018).

Ao associar as práticas de atenção plena com a alimentação, observa-se como a alimentação está intimamente associada a aspectos psicológicos, e que a alimentação consciente não se limita ao alimento, mas também as percepções e significados que os indivíduos atribuem a ele (Gidugu \& Jacobs, 2019). Isto contribui para redução do comer emocional, que é $\mathrm{o}$ ato de comer em excesso como resposta a emoções negativas e comumente observado em pessoas com sobrepeso (Frayn, et al., 2018), como observado em pacientes que realizaram cirurgia bariátrica que, embora a intervenção baseada em atenção plena não tenha auxiliado na perda de peso, houve redução do comer emocional (Chacko, et al., 2016) e da alimentação motivada por recompensas (Mason, Epel, Aschbacher, et al., 2016).

A aplicabilidade da atenção plena é vasta, podendo ser realizada em diferentes cenários e com diferentes grupos. Em estudo realizado com indivíduos com doença mental grave, no qual foi realizado um curso que associou a alimentação consciente com a educação nutricional, foi observado o desenvolvimento da consciência alimentar, diminuição do nível de distração e redução do comer emocional e excessivo entre os participantes (Gidugu \& Jacobs, 2019). Essa prática também impacta positivamente a saúde mental dos indivíduos, sendo eficaz na ansiedade, depressão e metacognição (Rogers, et al., 2017) e, uma vez contribuindo para redução dessas emoções negativas, auxiliará na redução do comer emocional e excessivo e, consequentemente, na perda e/ou manutenção do peso.

A atenção plena também contribui para a redução do consumo de doces e da glicemia de jejum (Mason, Epel, Kristeller, et al., 2016). A adoção dessa abordagem pode favorecer o cuidado de pacientes diabéticos, visto que possibilita que o indivíduo reflita sobre o seu padrão e comportamento alimentar, reduzindo o consumo excessivo e auxiliando em escolhas alimentares mais saudáveis, que atendam às suas necessidades de saúde e que favoreçam a autogestão da doença (Miller, 2017). Dessa forma, Mason, Epel, Kristeller et al. (2016) sugerem a utilização de práticas de atenção plena ao comer para redução da ingestão de doces e manutenção de níveis adequados de glicemia a longo prazo em adultos obesos.

Os benefícios dessa prática na redução do peso corporal podem se dar a longo prazo, como observado em diferentes estudos (Lucas, et al., 2017; Mason, Epel, Aschbacher, et al., 2016), sendo uma alternativa para o modelo tradicional utilizado para perda de peso. Dietas restritivas têm se mostrado uma prática ineficaz para perda de peso devido a elevada possibilidade de reganho de peso (Kortchmar, et al., 2018), apresentando resultados a curto prazo (Freire, 2020), enquanto a alimentação consciente promove a redução do peso a longo prazo (Dunn, et al., 2018), ao mesmo tempo em que trabalha aspectos psicológico do indivíduo, pela autoaceitação e atitudes de não julgamento (Palmeira, et al., 2017). Isto faz com que a promoção da atenção plena ao comer se torne uma melhor alternativa para pessoas que seguem dietas com restrições calóricas através da abordagem comportamental (Artiles, et al., 2019), contribuindo para uma maior consciência dos sinais de fome e saciedade, entre outros aspectos.

Tendo em vista que a obesidade é um problema de saúde pública global, caracterizada como uma doença crônica complexa e multifatorial (Montañez \& Mattiello, 2016), é importante a adoção de estratégias que visem a redução e/ou manutenção do peso como forma de promoção da saúde e prevenção de agravos, principalmente das doenças crônicas não transmissíveis. Para isso, a atenção plena ao comer e o aconselhamento nutricional são ações que apresentaram resultados positivos na perda e controle do peso (Artiles, et al., 2019), mas também no autogerenciamento da perda de peso (Hanson, et al., 2019) e, consequentemente, na promoção da saúde daquelas que a praticam. 


\section{Considerações Finais}

A prática de atenção plena associado ao aconselhamento nutricional se mostrou eficaz para promoção da consciência alimentar, contribuindo para redução do comer emocional e excessivo, adoção de padrões alimentares mais saudáveis, perda de peso, diminuição do auto estigma do peso, estimula o autorrespeito, a autocompaixão, contribui para autonomia do indivíduo frente ao processo de cuidado e traz melhorias para qualidade de vida.

Os benefícios dessa prática são inúmeros e, por contemplar diferentes dimensões da vida, vão além da alimentação contribuindo, por exemplo, para a saúde mental dos indivíduos. Além disso, pode ser direcionada para indivíduos com diferentes necessidades de saúde e que apresentem ou não sobrepeso/obesidade. Porém, é necessário a ampliação da divulgação destas informações acerca da prática da atenção plena, assim como a sua inserção e maior utilização nos serviços de saúde.

Mais estudos devem ser realizados utilizando essa prática, especialmente na atenção primária à saúde, tendo em vista a versatilidade de utilização em diferentes contextos e para diferentes públicos, e do seu baixo custo, eficácia e efetividade.

\section{Referências}

Artiles, R. A., Staub, K., Aldakak, L., Eppenberger, P., Rühli, F., \& Bender, N. (2019). Mindful eating and common diet programs lower body weight similarly: Systematic review and meta-analysis. Obesity Reviews, 20 (11), 1619-1627. 10.1111/obr.12918

Bal, U., Çelikbaş, Z., \& Batmaz, S. (2018). Association of mindfulness and impulsivity with obesity. Archives of Clinical Psychiatry (São Paulo), 45 (5), 130134. 10.1590/0101-60830000000173

Chacko, S. A., Yeh, G. Y., Davis, R. B., \& Wee, C. C. (2016). A mindfulness-based intervention to control weight after bariatric surgery: Preliminary results from a randomized controlled pilot trial. Complementary Therapies in Medicine, 28, 13-21. 10.1016/j.ctim.2016.07.001

Chung, S., Zhu, S., Friedmann, E., Kelleher, C., Kozlovsky, A., Macfarlane, K. W., Tkaczuk, K. H. R., Ryan, A. S., \& Griffith, K. A. (2016). Weight loss with mindful eating in African American women following treatment for breast cancer: a longitudinal study. Supportive Care in Cancer, 24 (4), $1875-1881$. $10.1007 / \mathrm{s} 00520-015-2984-2$

Dunn, C., Haubenreiser, M., Johnson, M., Nordby, K., Aggarwal, S., Myer, S., \& Thomas, C. (2018). Mindfulness Approaches and Weight Loss, Weight Maintenance, and Weight Regain. Current obesity reports, 7 (1), 37-49. 10.1007/s13679-018-0299-6

Frayn, M., Livshits, S., \& Knäuper, B. (2018). Emotional eating and weight regulation: A qualitative study of compensatory behaviors and concerns. Journal of Eating Disorders, 6 (1), 1-10. 10.1186/s40337-018-0210-6

Freire, R. (2020). Scientific evidence of diets for weight loss: Different macronutrient composition, intermittent fasting, and popular diets. Nutrition, 69, 110549. 10.1016/j.nut.2019.07.001

Gidugu, V., \& Jacobs, M. L. (2019). Empowering individuals with mental illness to develop healthy eating habits through mindful eating: results of a program evaluation. Psychology, Health \& Medicine, 24 (2), 177-186. 10.1080/13548506.2018.1516295

Hanson, P., Shuttlewood, E., Halder, L., Shah, N., Lam, F. T., Menon, V., \& Barber, T. M. (2019). Application of Mindfulness in a Tier 3 Obesity Service Improves Eating Behavior and Facilitates Successful Weight Loss. The Journal of Clinical Endocrinology \& Metabolism, 104 (3), 793-800. 10.1210/jc.201800578

Hirayama, M. S., Milani, D., Rodrigues, R. C. M., de Barros, N. F., \& Alexandre, N. M. C. (2014). A percepção de comportamentos relacionados à atenção plena e a versão brasileira do freiburg mindfulness inventory. Ciencia e Saude Coletiva, 19 (9), 3899-3914. 10.1590/1413-81232014199.12272013

Hussain, M., Egan, H., Keyte, R., \& Mantzios, M. (2021). Mindful Construal Reflections: Reducing Unhealthier Eating Choices. Mindfulness, 12 (7), 17571767. $10.1007 / \mathrm{s} 12671-021-01638-0$

Jordan, C. H., Wang, W., Donatoni, L., \& Meier, B. P. (2014). Mindful eating: Trait and state mindfulness predict healthier eating behavior. Personality and Individual Differences, 68, 107-111. 10.1016/j.paid.2014.04.013

Kortchmar, E., Merighi, M. A. B., Conz, C. A., De Jesus, M. C. P., \& De Oliveira, D. M. (2018). Weight regain after bariatric surgery: A phenomenological approach. ACTA Paulista de Enfermagem, 31 (4), 417-422. 10.1590/1982-0194201800058

Lucas, A. R., Focht, B. C., Cohn, D. E., Buckworth, J., \& Klatt, M. D. (2017). A Mindfulness-Based Lifestyle Intervention for Obese, Inactive Endometrial Cancer Survivors: A Feasibility Study. Integrative Cancer Therapies, 16 (3), 263-275. 10.1177/1534735416668257

Mason, A. E., Epel, E. S., Aschbacher, K., Lustig, R. H., Acree, M., Kristeller, J., Cohn, M., Dallman, M., Moran, P. J., Bacchetti, P., Laraia, B., Hecht, F. M., \& Daubenmier, J. (2016). Reduced reward-driven eating accounts for the impact of a mindfulness-based diet and exercise intervention on weight loss: Data from the SHINE randomized controlled trial. Appetite, 100, 86-93. 10.1016/j.appet.2016.02.009 
Research, Society and Development, v. 10, n. 16, e17101623003, 2021

(CC BY 4.0) | ISSN 2525-3409 | DOI: http://dx.doi.org/10.33448/rsd-v10i16.23003

Mason, A. E., Epel, E. S., Kristeller, J., Moran, P. J., Dallman, M., Lustig, R. H., Acree, M., Bacchetti, P., Laraia, B. A., Hecht, F. M., \& Daubenmier, J. (2016). Effects of a mindfulness-based intervention on mindful eating, sweets consumption, and fasting glucose levels in obese adults: data from the SHINE randomized controlled trial. Journal of Behavioral Medicine, 39 (2), 201-213. 10.1007/s10865-015-9692-8

Mendes, K. D. S., Silveira, R. C. de C. P., \& Galvão, C. M. (2008). Revisão integrativa: método de pesquisa para a incorporação de evidências na saúde e na enfermagem. Texto \& Contexto - Enfermagem, 17 (4), 758-764. 10.1590/S0104-07072008000400018

Miller, C. K. (2017). Mindful eating with diabetes. Diabetes Spectrum, 30 (2), 89-94. 10.2337/ds16-0039

Montañez, W. C., \& Mattiello, R. (2016). Obesidade: um grande desafio de saúde pública global. Ciência \& Saúde, 9 (3), $127.10 .15448 / 1983-$ 652X.2016.3.25844

O’Reilly, G. A., Cook, L., Spruijt-Metz, D., \& Black, D. S. (2014). Mindfulness-based interventions for obesity-related eating behaviours: a literature review. Obesity Reviews, 15 (6), 453-461. 10.1111/obr.12156

Oliveira, J., Oskinis, S., Santos, A. C. dos, \& Cordás, T. A. (2020). Existe uma relação entre autocompaixão e adição à comida em mulheres com comportamentos alimentares disfuncionais? Jornal Brasileiro de Psiquiatria, 69 (4), 211-219. 10.1590/0047-2085000000286

Özkan, N., \& Bilici, S. (2021). Are anthropometric measurements an indicator of intuitive and mindful eating? Eating and Weight Disorders, 26 (2), $639-648$. $10.1007 / \mathrm{s} 40519-020-00904-9$

Palmeira, L., Pinto-Gouveia, J., \& Cunha, M. (2017). Exploring the efficacy of an acceptance, mindfulness \& compassionate-based group intervention for women struggling with their weight (Kg-Free): A randomized controlled trial. Appetite, 112, 107-116. 10.1016/j.appet.2017.01.027

Rogers, J. M., Ferrari, M., Mosely, K., Lang, C. P., \& Brennan, L. (2017). Mindfulness-based interventions for adults who are overweight or obese: a metaanalysis of physical and psychological health outcomes. Obesity Reviews, 18 (1), 51-67. 10.1111/obr.12461

Souza, M. T. De, Silva, M. D. da, \& Carvalho, R. De. (2010). Integrative review: what is it? How to do it? Einstein (São Paulo), 8 (1), $102-106$. $10.1590 / \mathrm{s} 1679-45082010 \mathrm{rw} 1134$

Stites, S. D., Singletary, S. B., Menasha, A., Cooblall, C., Hantula, D., Axelrod, S., Figueredo, V. M., \& Phipps, E. J. (2015). Pre-ordering lunch at work. Results of the what to eat for lunch study. Appetite, 84, 88-97. 10.1016/j.appet.2014.10.005

Warren, J. M., Smith, N., \& Ashwell, M. (2017). A structured literature review on the role of mindfulness, mindful eating and intuitive eating in changing eating behaviours: Effectiveness and associated potential mechanisms. Nutrition Research Reviews, 30 (2), 272-283. 10.1017/S0954422417000154 